\title{
Costs and benefits of the MRSA Search and Destroy policy in a Dutch hospital
}

\author{
M. M. L. van Rijen • J. A. J. W. Kluytmans
}

Received: 11 February 2009 /Accepted: 26 June 2009 / Published online: 18 July 2009

(C) Springer-Verlag 2009

\begin{abstract}
The objective of this study was to determine the costs and benefits of the MRSA Search and Destroy policy in a Dutch hospital during 2001 through 2006. Variable costs included costs for isolation, contact tracing, treatment of carriers and closure of wards. Fixed costs were the costs for the building of isolation rooms and the salary of one full-time infection control practitioner. To determine the benefits of the Search and Destroy policy, the transmission rate during the study period was calculated. Furthermore, the number of cases of meticillin-resistant Staphylococcus aureus (MRSA) bacteraemia prevented was estimated, as well as its associated prevented costs and patient lives. The costs of the MRSA policy were estimated to be $€ 215,559$ a year, which equals $€ 5.54$ per admission. The daily isolation costs for MRSAsuspected and -positive hospitalised patients were $€ 95.59$ and $€ 436.62$, respectively. Application of the Search and Destroy policy resulted in a transmission rate of 0.30 and was estimated to prevent 36 cases of MRSA bacteraemia per year, resulting in annual savings of $€ 427,356$ for the hospital and ten lives per year (95\% confidence interval [CI] 8-14). In conclusion, application of the MRSA Search and Destroy
\end{abstract}

M. M. L. van Rijen ( $₫)$

Laboratory for Microbiology and Infection Control,

Amphia Hospital Breda,

Langendijk 75, P.O. Box 90157, 4800 RL Breda,

The Netherlands

e-mail: mvrijen@amphia.nl

J. A. J. W. Kluytmans

Laboratory for Microbiology and Infection Control, Amphia Hospital Breda,

P.O. Box 90158, 4800 RK Breda, The Netherlands

J. A. J. W. Kluytmans

Department of Medical Microbiology and Infection Control,

VU Medical Centre,

P.O. Box 7057, 1007 MB Amsterdam, The Netherlands policy in a hospital in a country with a low endemic MRSA incidence saves money and lives.

\section{Introduction}

Meticillin-resistant Staphylococcus aureus (MRSA) has become an increasingly important pathogen in hospitals worldwide and, recently, also in the community [1]. In the Netherlands and in Scandinavian countries, the percentage of $S$. aureus bacteraemia caused by MRSA is very low $(\leq 1 \%)$, contrary to other European countries that have reached percentages of up to $50 \%$ [2]. The low incidence in the Netherlands and Scandinavian countries is maintained by an active Search and Destroy (S\&D) policy, outlined in the national guidelines of the Dutch Working Party on Infection Prevention (WIP) [3]. The main aspects of the S\&D policy are the screening and isolation of patients considered at increased risk for the carriage of MRSA. This policy mainly affected persons that have been treated in a hospital abroad. However, since July 2006, a new group has been added, i.e. patients with exposure to pigs or veal calves $[4,5]$. Isolation is performed in a room with an anteroom, where health care workers (HCWs) must put on their personal protective equipment (PPE; mask, hat, gown and gloves). Because MRSA can be transmitted by air, an air pressure gradient ( $-15 \mathrm{Pascal}$ in the room, $-7.5 \mathrm{~Pa}$ in the anteroom and $0 \mathrm{~Pa}$ in the corridor) is required to prevent airflow (which may contain MRSA) from the room to the corridor. Carriers of MRSA (both patients and HCWs) are treated with antibiotics, which are described in the guidelines of the Dutch Working Party on Antibiotic Policy (SWAB) [6]. Up till now, few studies have estimated the costs and benefits of the S\&D policy [7, 8]. The objective of this study was to determine the costs and benefits of the 
S\&D policy in a large teaching hospital, the Amphia hospital, in a country with a low MRSA prevalence. The S\&D policy in the Amphia hospital is based entirely on the national MRSA guideline from the WIP [3]. The implementation of the guideline is controlled by the Inspection for Healthcare.

\section{Materials and methods}

\section{Setting}

This analysis was performed at the Amphia hospital, a teaching hospital with 1,370 beds. All medical specialties are present. The hospital is located in the south-western part of the Netherlands and serves a population of $\sim 440,000$ inhabitants. During the study period, on average, 38,943 patients were admitted annually to this hospital, with 282,585 patient days per year (mean numbers for the period 2001 through 2006).

\section{MRSA screening and confirmation}

Culture swabs were inoculated on a blood agar plate and in a broth enrichment. Culture plate and broth enrichment were incubated at $35-37^{\circ} \mathrm{C}$ overnight. After 1 day, the broth enrichment was inoculated on a blood culture plate and a mannitol salt agar plate with oxacillin $(2 \mathrm{mg} / \mathrm{l})$. Colonies which were suspected for $S$. aureus were tested by a latex agglutination test and the susceptibility for oxacillin was determined on a Mueller-Hinton agar plate with $2 \% \mathrm{NaCL}$. All strains were confirmed to be MRSA by polymerase chain reaction (PCR) for the nuc gene and the mecA gene. MRSA strains from all patients and HCWs were typed by the Dutch National Reference Centre (RIVM, Bilthoven, the Netherlands). This method was unchanged during the study period.

\section{Data collection}

The data of all patients and HCWs that were found to be carrying MRSA during the years 2001 through 2006 were prospectively recorded in a database. The following items were recorded: patient identification number, date of birth, date of first MRSA-positive culture, MRSA pulsed-field gel electrophoresis (PFGE) type, MRSA polymorphic X-region of the protein A gene (Spa) type, MRSA source, whether MRSA was found by targeted screening or by coincidence, number of screened contact patients and HCWs (both unprotected and protected contacts), number of secondary cases, number of days that MRSA-positive HCWs were suspended from work, whether MRSA treatment was given and, if so, whether MRSA was eradicated.
Estimation of costs for the hospital

The costs of the MRSA S\&D policy for the hospital in the years 2001 through 2006 were based on real data extracted from the above-mentioned data bank and other data (isolation database and registration forms) of the infection control department. First, costs were divided into variable and fixed costs. The method for the estimation of the variable costs is shown in Fig. 1. Cases were stratified based on the location of the initial finding (inpatients or outpatients clinic), if they were detected by targeted screening or as a coincidental finding and whether nosocomial spread had occurred. Costs for materials for PPE (gown, gloves, mask and hat), screening cultures, cleaning, salaries, missed patient days, MRSA eradication treatment and the building of pressure-controlled isolation rooms were requested from the financial department of the hospital. To calculate the costs for PPE, the number of isolation days and outpatient visits of MRSA-suspected and -positive patients were extracted from the isolation database of the infection control department. For inpatients, the mean number of HCWs and visitors that entered the room daily were extracted from the contact lists of the archives of the infection control department. Treatment costs were estimated based on the number of treatments given to the MRSA-positive patients and HCWs. The mean costs were $€ 100$ per treatment. Strain typing costs $(€ 100 /$ strain) were not included in the costs analysis, as this is done in a reference laboratory which is reimbursed by the government and, in this study, the costs for the hospital were estimated. The fixed costs were divided into costs for the building of pressure-controlled isolation rooms and the salary of one full-time infection control practitioner. The costs of the microbiologist, who acts as the infection control physician as well, were included in the culture costs. Ten percent was added to the total costs for overheads and for costs that could not be estimated, for example, costs for disposables that had to be thrown away and for additional HCWs that were asked to work when an MRSA-positive patient was present on their ward.

Furthermore, the additional daily isolation costs for isolated patients and additional costs for unexpected MRSA-positive cases were estimated. Treatment costs were excluded in these analyses. To estimate the daily isolation costs for MRSA-positive and -suspected patients, the total variable isolation costs were divided by the total number of patients that had been appropriately isolated. The mean length of stay for MRSA-positive and -suspected patients was calculated and used to estimate the daily isolation costs. Fixed costs for building isolation rooms were divided by the total number of isolation days and added to the mean daily isolation costs. To estimate the additional costs that have to be made for patients and HCWs that were 
Fig. 1 Estimation of the variable costs of the Search and Destroy (S\&D) policy

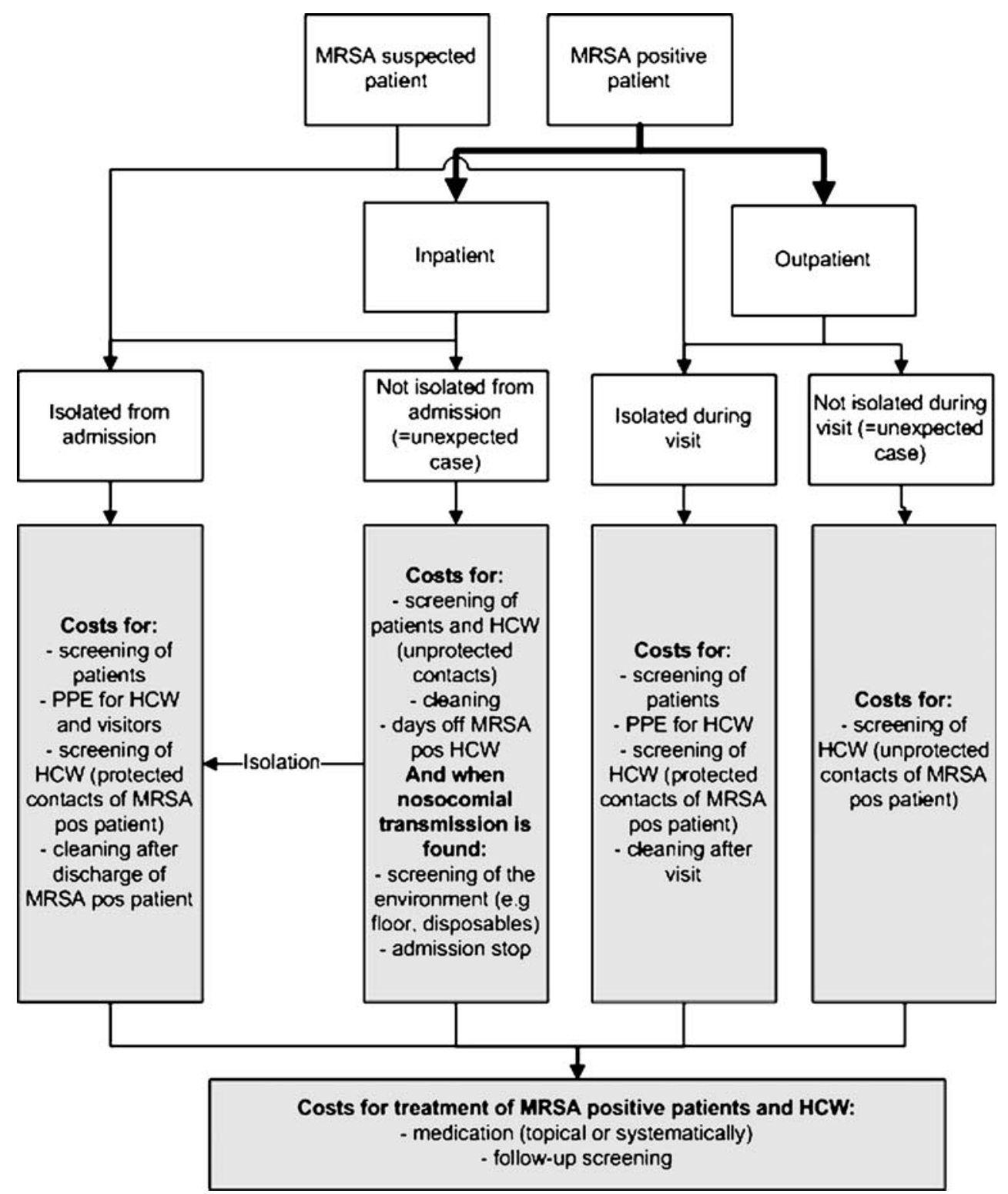

unexpectedly found to carry MRSA, the total variable costs for unexpected MRSA cases were divided by the number of patients and HCWs that were found to be MRSA-positive by coincidence. These additional costs were estimated for both the cases with and without nosocomial transmission.

\section{Estimation of benefits}

To estimate the effect of the S\&D policy in our hospital, the transmission rate (secondary cases/index cases) during the study period was calculated. Secondary cases were cases of patients and HCWs who were colonised by MRSA due to nosocomial transmission. When an MRSA-positive case found in the hospital could be linked in time (overlap in the dates of patient days with a maximal interval of 30 days) and place (overlap in wards, including adjacent wards) to an index patient or HCW and the MRSA strains were indistinguishable to the strain of the index, the source of the case was classified as nosocomial transmission. When no link in time and place could be found, the case was

Furthermore, the benefits of the situation under application of the S\&D policy was compared to a situation in which the Netherlands would have never applied the S\&D policy. Therefore, the mean annual number of patients with a nosocomial $S$. aureus bacteraemia that could be prevented and its associated saved costs and the number of patient lives that could be saved by application of the S\&D policy was estimated. This was based on the annual number of patients with a nosocomial $S$. aureus bacteraemia found in the Amphia hospital. Patients suffering from a $S$. aureus bacteraemia were identified in the laboratory information system. Data were available for the years after the implementation of this information system, i.e. 2004 classified as an index case. 
Table 1 Costs of the MRSA Search and Destroy policy in 2001 through 2006

Variable costs:

1. Isolation costs

MRSA-suspected patients (324 admissions)

PPE (gown, gloves, mask, hat for HCWs

and visitors

Screening of patients

$6(=$ mean contact list $)+5 /$ day

1,446 isolation days

$324 \times 4$ cultures $=1,296$

Subtotal of isolation costs for

MRSA-suspected patients

MRSA-positive patients (90 admissions)

PPE for HCWs and visitors

Screening of patients

Screening of HCWs (protected contacts

(= with PPE))

Cleaning after discharge

Subtotal of isolation costs for

MRSA-positive patients

Costs of outpatients clinic visits

(MRSA-positive and -suspected patients)

PPE for HCWs

Screening of HCWs (protected contacts)

Cleaning after visit

Screening of patients

Subtotal of isolation costs in the outpatients clinic

Subtotal of isolation costs

2. Costs of coincidental findings in the inpatients clinic without nosocomial spreading

Screening of patients (unprotected contacts)

Screening of HCWs (unprotected contacts)

Cleaning

Day offs of MRSA-positive HCWs

(job taken into account)

Subtotal of costs

3. Costs of coincidental findings in the inpatients clinic with nosocomial spreading

Screening of patients (unprotected contacts)

Screening of HCWs (unprotected contacts)

Screening of the environment

(e.g. floor, disposables)

Cleaning

Admission halt in outbreak situation

Day offs of MRSA-positive HCWs

(job taken intoaccount)

Subtotal of costs

\section{Costs of coincidental findings}

in the outpatientsclinic

Screening of HCWs (unprotected contacts)

Screening of patients (when HCWs is positive)

Cleaning

Subtotal of costs

5. Treatment costs
$6(=$ mean contact list $)+5 /$ day

644 isolation days

$90 \times 4$ cultures $=360$

$84 \mathrm{HCWs}$ (= mean contact list $) \times$

90 admissions $=7,560$

$90 \mathrm{~h}$

$936 / 6$ year

1,872

1,872

936

$936 \times 4$ cultures $=3,744$

133 days

2 outbreaks (14 patients and $9 \mathrm{HCWs})$

360 cultures

777 cultures

198 cultures

$257 \mathrm{~h}$

55 days

215 days

16 patients

211

0

0

12 HCWs with 14 treatments

32 patients with 44 treatments
$1.86 /$ set

29/culture

$50 / \mathrm{h}$

29/culture

29/culture

29/culture

$50 / \mathrm{h}$

90/day

$29,585.16$

$37,584.00$

$67,169.16$

$13,176.24$

$10,440.00$

$219,240.00$

$4,500.00$

$\mathbf{2 4 7 , 3 5 6 . 2 4}$

$3,481.92$

$54,288.00$

$46,800.00$

$108,576.00$

213,145.92

$\mathbf{5 2 7 , 6 7 1 . 3 2}$

$36,656.00$

$35,554.00$

$1,350.00$

$11,970.00$

$85,530.00$

29/culture

$10,440.00$

29/culture

$22,533.00$

$5,742.00$

$12,850.00$

$50 / \mathrm{h}$

$21,175.00$

$385 /$ day at ward

$19,350.00$

92,090.00

29/culture

6,119.00

0

0

6,119.00 
Table 1 (continued)

\begin{tabular}{|c|c|c|c|}
\hline & Number & Costs $(€)$ & $\begin{array}{l}2001-2006 \\
\text { total costs }(€)\end{array}$ \\
\hline Treatment (topical or systemically) & 58 treatments & $100 /$ treatment & 5,800 \\
\hline $\begin{array}{l}\text { Follow-up screening of treated } \\
\text { patients and HCWs }\end{array}$ & 1,524 cultures & 29/culture & $44,196.00$ \\
\hline Subtotal of costs & & & $49,996.00$ \\
\hline \multicolumn{4}{|l|}{ Fixed costs: } \\
\hline 1. Salary of infection control practitioner ( $1 \mathrm{fte})$ & $1 \mathrm{fte}$ & $51,661.5 /$ year & $309,969.00$ \\
\hline $\begin{array}{l}\text { 2. Building isolations rooms } \\
\text { (debit from } 20 \text { years) }\end{array}$ & 16 rooms & $21,750 /$ room & $104,400.00$ \\
\hline Subtotal of costs & & & $414,369.00$ \\
\hline Calculated costs from 2001 until 2006 & & & $1,175,775.32$ \\
\hline Overheads: $10 \%$ & & & $117,577.53$ \\
\hline Total costs from 2001 until 2006 & & & $1,293,352.52$ \\
\hline Costs per year & & & $215,558.81$ \\
\hline
\end{tabular}

through 2006. Each first S. aureus-positive blood sample of a patient was included; further positive samples were excluded. Samples taken from patients at the dialysis department were excluded because, in this patient population, it is difficult to determine whether the bacteraemia was acquired in the hospital or in the community. Bacteraemia were defined to be nosocomial when the first positive blood culture was sampled more than two days after admission. To estimate the number of lives that could be saved, the mortality rate during hospital stay was determined in patients with a nosocomial $S$. aureus bacteraemia. The assumption was made that, if no control measures would have been implemented, $50 \%$ of the nosocomial $S$. aureus bacteraemias would have been caused by MRSA, as is the case in many countries that do not apply an MRSA S\&D policy [2]. The introduction of MRSA has been shown to increase the absolute number of cases of nosocomial $S$. aureus bacteraemia, as has been shown in the UK $[9,10]$. So instead of replacement, MRSA adds to the existing burden of disease. Associated costs were based on the results of Cosgrove et al., who estimated the median hospital costs for patients with MRSA bacteraemia to be $€ 11,871(\$ 14,655)$ [11]. This calculation was based on the mean Dollar-Euro exchange rate of 2005, the year in which the study of Cosgrove et al. was published [11]. In our estimation of benefits, no difference in mortality between bacteraemia due to MRSA or meticillin-susceptible $S$. aureus) (MSSA) was taken into account.

Table 2 Mean additional costs per MRSA case and additional costs per isolation day during the period 2001 through 2006

\begin{tabular}{|c|c|c|c|c|c|c|c|}
\hline & $n$ & $\begin{array}{l}\text { Isolation } \\
\text { days }\end{array}$ & $\begin{array}{l}\text { Mean total variable } \\
\text { costs }(€) / \text { case }\end{array}$ & $\begin{array}{l}\text { Mean length } \\
\text { of stay }\end{array}$ & $\begin{array}{l}\text { Mean variable costs } \\
(€) / \text { isolation day }\end{array}$ & $\begin{array}{l}\text { Mean fixed costs } \\
(€) / \text { isolation day }\end{array}$ & $\begin{array}{l}\text { Total costs }(€) \\
\text { isolation day }\end{array}$ \\
\hline \multicolumn{8}{|l|}{ Inpatients clinic } \\
\hline MRSA-suspected patient in isolation & 324 & 1,446 & 207.31 & 4.5 & 46.07 & 49.52 & 95.59 \\
\hline MRSA-positive patient in isolation & 90 & 644 & $2,748.40$ & 7.1 & 387.10 & 49.52 & 436.62 \\
\hline $\begin{array}{l}\text { Coincidental finding without } \\
\text { nosocomial spread }^{1}\end{array}$ & 31 & NA & $2,759.03$ & NA & NA & NA & NA \\
\hline Outbreak situation (two outbreaks) $^{1}$ & 23 & NA & $4,003.91$ & NA & NA & NA & NA \\
\hline \multicolumn{8}{|l|}{ Outpatients clinic } \\
\hline $\begin{array}{l}\text { MRSA-positive/-suspected } \\
\text { patient in isolation }{ }^{2}\end{array}$ & 936 & NA & 227.72 & NA & NA & NA & NA \\
\hline $\begin{array}{l}\text { Coincidental finding without } \\
\text { nosocomial spread }\end{array}$ & 16 & NA & 382.44 & NA & NA & NA & NA \\
\hline
\end{tabular}


Fig. 2 Costs of the different items of the MRSA S\&Destroy policy in 2001 through 2006. ICP $=$ infection control practitioner

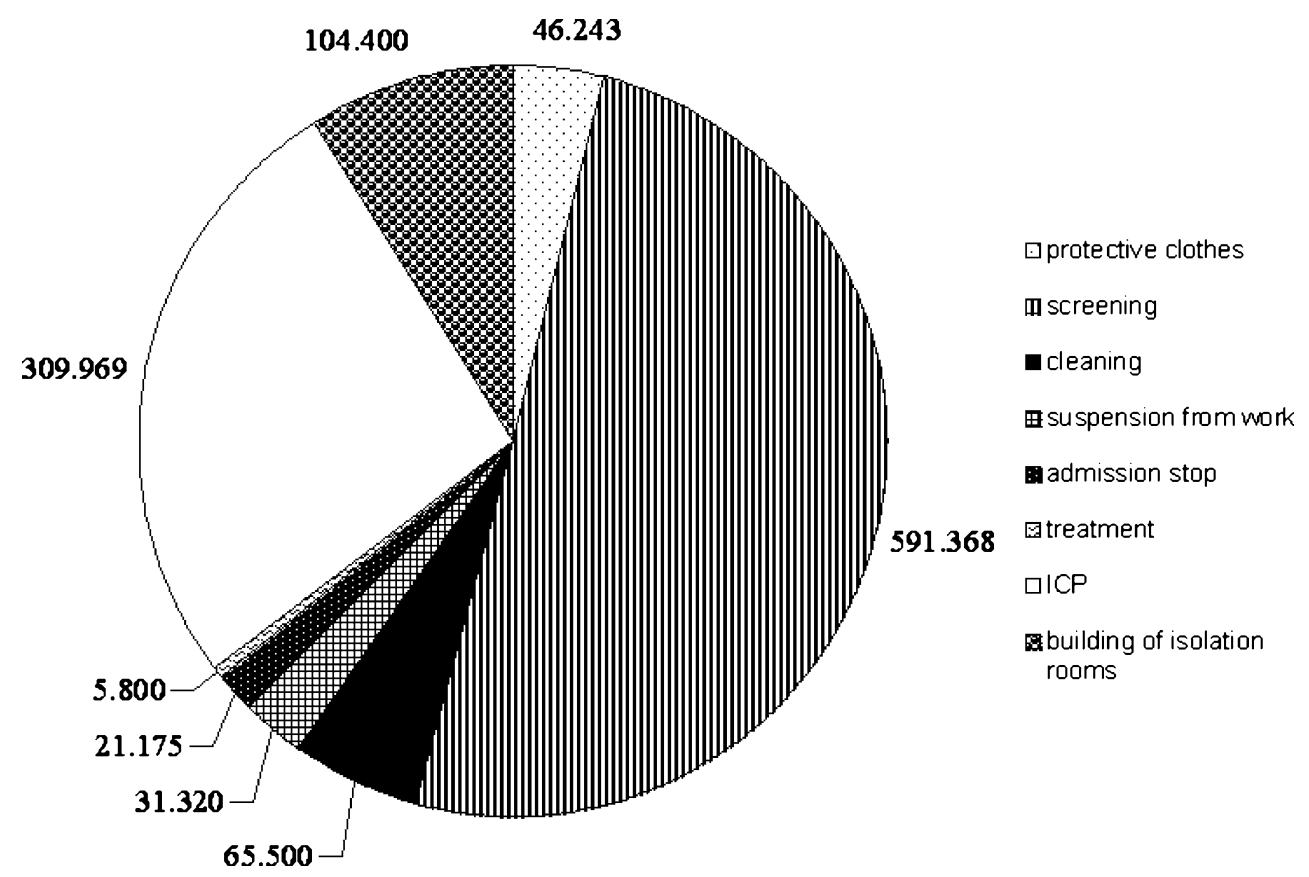

\section{Results}

Costs of the MRSA policy

The total costs of the MRSA policy in the years 2001 through 2006 were calculated to be $€ 1,293,353$. This is, on average, €215,559 per year. The costs are specified in Table 1.

The mean variable costs per MRSA-positive or -suspected case and costs per isolation day are shown in Table 2. Figure 2 shows the costs for the separate items of the S\&D policy. The main costs are caused by costs for screening and the salary of one full-time infection control practitioner.

Benefits due to the MRSA policy

Two outbreaks occurred during the study period, both caused by the same MRSA type (PFGE 55, Spa t003). The first patient was a patient who had been transferred from a hospital in Turkey. Unfortunately, this patient was not asked whether he belonged to an MRSA risk category on admission and was, therefore, not isolated until MRSA was found in a clinical culture and spread had occurred. Thirteen patients and nine HCWs were colonised by nosocomial transmission during these two outbreaks. The transmission rate during the study period was 0.30 (22 secondary cases/73 index cases).

In 2004 through 2006, the total number of patients that developed a nosocomial bacteraemia caused by $S$. aureus was 109 (mean $36 \pm 10$ per year), resulting in an incidence density of 1.3/10,000 patient days (109/833,716). All of these $S$. aureus strains were susceptible for meticillin.
Thirty-one of these patients (28.4\%) died during their hospital stay (mean of ten per year). Based on the assumption of $50 \%$ MRSA prevalence caused entirely by addition, 36 additional nosocomial bacteraemia would have been caused by MRSA each year when no control measures had been taken. Based on Cosgrove et al., the hospital costs for these 36 MRSA bacteraemia would have been $€ 427,356$ [11]. With comparable MRSA and MSSA mortality rates, this would have resulted in ten additional deaths $(95 \%$ confidence interval [CI] 8-14).

\section{Discussion}

Estimated costs

The costs of the S\&D policy were estimated to be $€ 215,559$ per year, equivalent to $€ 5.54$ per admission or $€ 0.76$ per patient day. This equals $0.08 \%$ of the hospital budget. The estimated costs are comparable to the costs as estimated by Vriens et al. [7]. The annual costs of the MRSA policy in the University Medical Centre Utrecht during the years 1991 through 2000 were estimated to be $€ 280,000$. Recently, Nulens et al. estimated the costs for pro-active searching for MRSA in the University Hospital Maastricht to be $€ 1,383,200$ per year [8]. This higher estimate is mainly caused by more screening tests due to the extension of the risk period from 2 to 6 months and the higher costs per isolation day, i.e. costs for extra $\mathrm{HCWs}$, disposables and additional room cleaning. In our hospital, almost no additional HCWs were asked to work, a stock of disposables for $24 \mathrm{~h}$ only was used and additional room cleaning was only required after discharge of the patient instead of 
every day. Apparently, this approach is effective as well, and is associated with much lower costs. In general, the costs estimated for the Amphia hospital are representative for all other Dutch hospitals, because they all follow the S\&D policy described in the WIP guideline [3]. Although there may be variations between hospitals in the implementation of the guideline, these are minor.

This is the first study that estimated the additional costs per MRSA-suspected or -positive case (Table 2). The daily incremental costs for hospitalised patients in isolation were relatively low, i.e. $€ 436.62$ for MRSA-positive patients and $€ 95.59$ for MRSA-suspected patients. This difference can be explained by the absence of screening of HCWs after contact with MRSA-suspected patients. However, relatively high additional costs have to be made for coincidental findings who were not in isolation from the very start, i.e. $€ 2,759.03$ for cases without nosocomial transmission and $€ 4,003.91$ for cases with nosocomial transmission. It confirms the importance to identify persons at risk for MRSA carriage and to take control measures as soon as possible.

In the Amphia hospital, the number of MRSA-positive persons increased during this study period due to the emergence of non-typable MRSA (NT-MRSA) [4]. This type is related to a reservoir in pigs and veal calves [5]. As this reservoir is now clearly established and is not likely to decrease in the future, this will result in higher costs for the MRSA policy, especially for the variable costs. In addition, the costs described in this article are costs for an MRSA S\&D policy in a country with low endemic MRSA levels. Costs that have to be made in a high prevalence setting will be much higher, because, in that setting, it is impossible to designate patients to a specific MRSA risk category. This will require more extensive screening. Also, more isolation rooms, PPE and MRSA eradication treatment are needed. The best way to handle this is probably a stepwise implementation. For example, the mathematical model of Bootsma et al. showed that, starting with a limited number of control measures (e.g. screening, precautionary isolation) will reduce the MRSA rates in the hospital [12]. Based on this model, it is estimated that application of the full S\&D policy in a high endemic setting will reduce endemic prevalence levels to $<1 \%$ within 6 years. However, the screening of contact patients (with precautionary isolation) of an index case will take, on average, 8 years to reach endemic levels $<1 \%$.

\section{Estimated benefits}

During the study period, hardly any nosocomial transmissions occurred (transmission rate of 0.30 ), which shows the effectiveness of the S\&D policy in a country with a low endemic MRSA incidence. Furthermore, the saving in costs for the hospital and the number of patient lives saved based on the prevention of MRSA bacteraemia was calculated to estimate the benefits for the Amphia hospital. This is extremely difficult, since it is not possible to determine accurately what would have happened if no control measures had been implemented in the Dutch situation. Therefore, it has to be based upon assumptions that try to translate the observed situation in countries with high rates of MRSA to the countries with low rates. In countries that did not implement the S\&D policy, the number of cases of bacteraemia caused by $S$. aureus increased when MRSA emerged [10]. So MRSA added to the existing burden of staphylococcal disease. If no S\&D policy had been implemented in our hospital, it was estimated that, annually, 36 cases of MRSA bacteraemia would be added to the present 36 cases of MSSA bacteraemia. The S\&D approach prevents these events and this was estimated to result in an annual saving of $€ 211,797$ (saved costs based on prevented MRSA bacteraemia - costs for the application of the S\&D policy $=€ 427,356-€ 215,559)$ for the hospital and of 10 (95\% CI 8-14) patient lives. This is probably an underestimation because of two reasons. First, there are indications that there is a difference in the mortality rate between bacteraemia caused by MRSA and MSSA [10, 13]. The meta-analysis of Cosgrove et al. showed a significantly higher mortality rate due to MRSA bacteraemia than due to MSSA (odds ratio [OR] 1.88, 95\% CI 1.33-2.69). Because this increased mortality due to MRSA is under discussion, we did not take it into account for our estimation of the benefits. Second, this estimation focussed on bacteraemia only and not on other infections caused by $S$. aureus. In the USA, the mortality rate due to MRSA invasive infections was estimated to be $6.3 / 100,000$ inhabitants in 2005 [14]. The Amphia hospital serves a population of $\sim 440,000$ inhabitants. In accordance with the situation in the USA, when no S\&D policy would have been applied in the Amphia hospital, 27 deaths due to invasive MRSA infection would have occurred in 2005. This is higher than the upper range of the confidence interval that we estimated. Therefore, we consider this to be a conservative estimate.

In conclusion, application of the MRSA S\&D policy in a hospital in a country with a low endemic MRSA incidence saves money and lives.

\section{References}

1. Kluytmans-VandenBergh MFQ, Kluytmans JAJW (2006) Community-acquired methicillin-resistant Staphylococcus aureus: current perspectives. Clin Microbiol Infect 12(Suppl 1):9-15

2. Tiemersma EW, Monnet DL, Bruinsma N, Skov R, Monen JCM, Grundmann H (2005) Staphylococcus aureus bacteremia, Europe. Emerg Infect Dis 11:1798-1799 
3. Dutch Working Party on Infection Prevention (WIP) (2007) MRSA hospital. Available online at: http://www.wip.nl/UK/ free_content/Richtlijnen/MRSA\%20hospital.pdf

4. van Rijen MML, Van Keulen PH, Kluytmans JAJW (2008) Increase in a Dutch hospital of methicillin-resistant Staphylococcus aureus related to animal farming. Clin Infect Dis 46(2):261263

5. van Loo I, Huijsdens X, Tiemersma E, de Neeling A, van de Sande-Bruinsma N, Beaujean D, Voss A, Kluytmans J (2007) Emergence of methicillin-resistant Staphylococcus aureus of animal origin in humans. Emerg Infect Dis 13:1834-1839

6. Wertheim HF, Ammerlaan HS, Bonten MJ, van den Broek PJ, Troelstra A, Vandenbroucke-Grauls CM, Vos MC, Voss A, Nouwen JL, Kluytmans JA (2008) Optimisation of the antibiotic policy in the Netherlands. XII. The SWAB guideline for antimicrobial eradication of MRSA in carriers. Ned Tijdschr Geneeskd 152(49):2667-2671

7. Vriens M, Blok H, Fluit A, Troelstra A, van der Werken C, Verhoef J (2002) Costs associated with a strict policy to eradicate methicillin-resistant Staphylococcus aureus in a Dutch university medical center: a 10-year survey. Eur J Clin Microbiol Infect Dis 21:782-786

8. Nulens E, Broex E, Ament A, Deurenberg RH, Smeets E, Scheres J, van Tiel FH, Gordts B, Stobberingh EE (2008) Cost of the meticillin-resistant Staphylococcus aureus search and destroy policy in a Dutch university hospital. J Hosp Infect 68(4):301-307
9. Griffiths C, Lamagni TL, Crowcroft NS, Duckworth G, Rooney C (2004) Trends in MRSA in England and Wales: analysis of morbidity and mortality data for 1993-2002. Health Stat Q 21:15-22

10. Wyllie DH, Crook DW, Peto TEA (2006) Mortality after Staphylococcus aureus bacteraemia in two hospitals in Oxfordshire, 1997-2003: cohort study. BMJ 333(7562):281

11. Cosgrove SE, Qi Y, Kaye KS, Harbarth S, Karchmer AW, Carmeli Y (2005) The impact of methicillin resistance in Staphylococcus aureus bacteremia on patient outcomes: mortality, length of stay, and hospital charges. Infect Control Hosp Epidemiol 26(2):166174

12. Bootsma MC, Diekmann O, Bonten MJ (2006) Controlling methicillin-resistant Staphylococcus aureus: quantifying the effects of interventions and rapid diagnostic testing. Proc Natl Acad Sci USA 103(14):5620-5625

13. Cosgrove SE, Sakoulas G, Perencevich EN, Schwaber MJ, Karchmer AW, Carmeli Y (2003) Comparison of mortality associated with methicillin-resistant and methicillin-susceptible Staphylococcus aureus bacteremia: a meta-analysis. Clin Infect Dis 36:53-59

14. Klevens RM, Morrison MA, Nadle J, Petit S, Gershman K, Ray S, Harrison LH, Lynfield R, Dumyati G, Townes JM, Craig AS, Zell ER, Fosheim GE, McDougal LK, Carey RB, Fridkin SK; Active Bacterial Core surveillance (ABCs) MRSA Investigators (2007) Invasive methicillin-resistant Staphylococcus aureus infections in the United States. JAMA 298(15):1763-1771 